\title{
Amerikanische Konstruktionen des Krieges und der Selbst- verteidigung nach dem 11. September 2001
}

In vielen politikwissenschaftlichen und völkerrechtlichen Analysen wird der amerikanische „Krieg gegen den Terror" als eine neue Art von Krieg angesehen, der sowohl das Gewaltverbot der Charta der Vereinten Nationen als auch das Recht auf Selbstverteidigung nach einem Angriff von außen berührt. Eine Diskursanalyse der Konstruktionen des Kriegsbegriffs und der Selbstverteidigung seit dem 11. September 2001 durch die USRegierung zeigt, dass die amerikanische Kritik an der gängigen Auslegung des Selbstverteidigungsrechts nicht in eine positive Rechtsbehauptung umgesetzt wurde. Stattdessen wurde der in der Tradition der philosophischen Lehre vom Krieg zentrale Begriff ,, Gerechtigkeit" in unterschiedlicher Weise moralisch, evangelikal oder juristisch gebraucht und damit eine große Unsicherheit in der völkerrechtlichen Diskussion erzeugt. Auf keinen Fall eignete sich der Diskurs jedoch zur Schaffung neuen Gewohnheitsrechts nach dem 11. September oder zur Reinterpretation des völkerrechtlichen Gewaltverbots.

\section{Inhalt}

1. Einleitung

2. Zum theoretischen Verständnis: Konstruktion und Diskurs 358

3. Kriegs- und Bedrohungskonstruktionen 360

4. Konstruktionen des jus ad bellum $\quad 365$

5. Konstruktion, Krieg und Selbstverteidigung $\quad 372$

Literatur

\section{Einleitung}

Seit dem 11. September 2001 führten die USA im Rahmen ihrer Antiterror-Strategie zwei Kriege. In beiden Fällen wurden die zuvor formulierten Kriegsziele nicht erreicht, beide Male war die Zahl ziviler Opfer sehr hoch und beide Kriege wurden ohne feste völkerrechtliche oder moralische Grundlage geführt. Argumentativ wurden sie mit einem präzedenzlosen Aufwand vorbereitet, begleitet und legitimiert. Dies war nötig, da es sich nach 
Ansicht vieler Beobachter um eine „,neue Art von Krieg“"1 handelte, der sowohl traditionelle Bedeutungen des jus ad bellum wie auch des jus in bello infrage stellte.

Um die Konstruktion eines neuen Kriegsbegriffs und die Rekonstruktion des völkerrechtlichen Gewaltverbots durch die amerikanische Regierung nach den Anschlägen des 11. September geht es in diesem Beitrag. Im Mittelpunkt steht die Analyse von Diskursen über Krieg und völkerrechtliche Regeln der Gewaltanwendung. Dazu wird zunächst das theoretische Verständnis von sozialer Konstruktion und Diskursanalyse entwickelt, um auf dieser Grundlage erstens die diskursive Entwicklung eines neuen Kriegsbegriffs und zweitens die Reinterpretation gängiger Regeln der Gewaltanwendung zu analysieren. Damit soll ein Beitrag zur Diskussion um den theoretischen Zusammenhang von Sprache, Diskurs und Macht einerseits sowie zur Fortentwicklung des Völkerrechts nach dem 11. September 2001 andererseits geleistet werden.

Das Hauptargument lautet, dass durch die Vermischung - mitunter obsoleter - juristischer, evangelikaler und moralischer Kategorien durch die amerikanische Regierung eine völkerrechtliche Anomie eingetreten ist. Auf keinen Fall, so die These, eignet sich der „Krieg gegen den Terror“ als Präzedenzfall für die Aufweichung des Gewaltverbots der Charta der Vereinten Nationen und eine Neuregelung des Rechts auf Selbstverteidigung. ${ }^{2}$

\section{Zum theoretischen Verständnis: Konstruktion und Diskurs}

Es waren vor allem poststrukturalistische und kritische Theorien, die der Diskursanalyse in den vergangenen zwei Jahrzehnten einen festen Platz innerhalb der Disziplin der Internationalen Beziehungen (IB) verschafften. ${ }^{3}$ Bedeutende Einflüsse kamen dabei aus anderen Disziplinen. Dieser Beitrag rekurriert insbesondere auf die Methode der kritischen Diskursanalyse Norman Faircloughs, der politische Kommunikation als Typ sozialer Praxis konzeptualisiert (Fairclough 1989, 1992, 2003). Nach Fairclough ist Diskurs ein Element des sozialen Lebens, das in dialektischer Weise mit anderen Elementen verbunden ist (2003: 214 f.). Diskurs konstituiert soziale Beziehungen; der Begriff umfasst im Wesentlichen drei Dimensionen: Wissen, soziale Beziehungen und soziale Identität (Fairclough 1992: 8). Einzelne Texte sind nach diesem Diskursverständnis feinfühlige Barometer sozialer Prozesse, sie weisen auf sozialen Wandel und soziale Unterschiede hin (Fairclough 1999: 204). Ziel der Diskursanalyse ist es, die Verbindungslinien zwischen Sprache sowie sozialen Praktiken und Strukturen aufzudecken.

Die kritische Diskursanalyse von Fairclough geht im Einklang mit der Theorie von Laclau und Mouffe davon aus, dass diskursive Praktiken niemals neutral sind, sondern zur Herstellung und Reproduktion von ungleichen Machtverhältnissen beitragen. Am besten funktioniert dieser Mechanismus in Zeiten von identitärer Fragmentierung und

1 McInnes 2003: 173; Lawler 2002: 154; aus juristischer Perspektive z. B. Tomuschat 2001 und Seidel 2003.

2 Ähnlich Thürer 2003; Bothe 2003; Seidel 2003.

3 Z. B. Doty 1996; Larsen 1997; Waever 1998; Diez 1999; Milliken 1999; Jackson 2005; Nabers 2005. 
Unbestimmtheit. ${ }^{4}$ Insbesondere Traumata (wie die Anschläge vom 11. September) können Ausdruck einer solchen Situation sein. Sie beziehen sich auf Ereignisse, die so schockierend sind, dass sie unsere alltäglichen Erwartungen über die Welt ernsthaft gefährden (Edkins 2002: 246 f.; Brockhaus 2002). Traumata überfordern die Verarbeitungsfähigkeiten eines einzelnen Menschen und ziehen das individuelle Vertrauen in die Gestaltung des eigenen Lebens in Zweifel. Gerade diese Situation eröffnet völlig neue Interpretationsspielräume, die in der Konfiguration neuer kollektiver Bedeutungsmuster münden.

Methodisch kann die Bedeutung der Redefinition sozialer Beziehungen nur durch die Analyse miteinander verbundener Texte erfasst werden. Differenzen werden durch Diskurse konstituiert; sie konstruieren die Bedeutung der Welt, und ,every object is constituted as an object of discourse" (Laclau/Mouffe 1985: 107). Diese Denkweise bedeutet einen Bruch mit der künstlichen Dichotomie einer diskursiven und extra-diskursiven Welt. Gleichzeitig bedeutet sie eine Abkehr von der positivistischen Opposition zwischen Denken und Realität. Die Dimension der Verbundenheit von Texten, ihrer Intertextualität, der Inkorporation and Interpretation anderer Texte, der Allusion und des Dialogs stehen im Mittelpunkt der Fairclough'schen Methodik (Fairclough 2003: 36, 47). Die intertextuelle Analyse beschäftigt sich implizit mit der Verbundenheit von Text und Gesellschaft und richtet unser Augenmerk damit auf die Frage, wie Texte Gesellschaften transformieren (Fairclough 1999: 184 f.). Intertextualität bedeutet also, dass sich jeder Diskurs auf andere Diskurse bezieht, die vorher, außerhalb und unabhängig schon existieren. Einzelne semantische Elemente sind bereits in früheren Zusammenhängen vorgekommen; durch bestimmte Inferenzen, Konnotationen und semantische Merkmale können sie auch in anderen $\mathrm{Zu}$ sammenhängen wieder vorkommen. Ein Beispiel ist der englische Begriff „evil“ (böse), der im amerikanischen Antiterror-Diskurs häufig benutzt wird, um den Feind zu etikettieren. Er stammt jedoch aus einem ganz anderen Diskurs, einem christlichen, und erfordert zu seiner Einordnung den Rückgriff auf das damit verbundene Vorverständnis. Der Begriff ist so in den Interdiskurs eingeschrieben und steht quasi als in einem bestimmten Zusammenhang aktualisierbares Element zur Verfügung.

Ein erster Analyseschritt bezieht sich auf die Identifizierung der Sprecher eines Textes sowie anderer Texte, auf die Bezug genommen wird. Darüber hinaus geht es um die Aufdeckung der wichtigsten Annahmen eines Textes. Annahmen umfassen implizite Unterstellungen, logische Implikationen und Folgerungen (Fairclough 2003: 40). Sie lassen sich grob in drei Typen einteilen: Realitätsannahmen (Was existiert?), Aussageannahmen (Was ist? Was könnte sein? Was wird sein?) und Wertannahmen (Was ist gut oder wünschenswert?) (Fairclough 2003: 55 f.).

Das Analyseziel besteht in der Aufdeckung des Prozesses, in dem partikulare zu universellen Diskursen werden. Die Wiederholung bestimmter Argumentationsmuster macht es dem Wissenschaftler möglich, die der Handlung zugrunde liegende Logik zu isolieren. Eine Analyse kann als abgeschlossen gelten, wenn durch die Auswahl neuer Texte keine abweichenden Standpunkte mehr identifiziert werden können. Dem Verfahren des ,hermeneutischen Zirkels“" vergleichbar, wird durch Prüfung und Korrektur bestimmter Interpretationsangebote eine „Lesart“ identifiziert, die die sozialen Praktiken der Diskursteilnehmer

4 Laclau/Mouffe 1985: 13; Laclau 1977: 103, 1990: 64. 
als kohärent erscheinen lässt (Mayer 2003: 65 f.). In diesem Stadium wird sogar der Beweis der wissenschaftlichen Konsistenz der Diskursanalyse möglich, da die angebotene Interpretation immer wieder überprüft und überarbeitet werden kann (Milliken 1999: 234 f.; Titscher u. a. 2000: 41 f.). Schließlich können bestimmte Deutungsmuster und Argumentationen, die zur Stabilisierung bzw. Destabilisierung von Akteurskonstellationen führen, abstrakt gefasst werden, um in weiterführenden Studien einen Vergleich zu anderen Politikfeldern möglich zu machen. Mit diesem Diskursverständnis sollen nun die zwei hervorstechenden diskursiven Konstruktionen des amerikanischen Antiterror-Diskurses untersucht werden: die Konstruktion eines neuen Kriegsbegriffs und die Auslegung von völkerrechtlichem Gewaltverbot und Selbstverteidigungsrecht (jus ad bellum).

\section{Kriegs- und Bedrohungskonstruktionen}

Akte politischer Gewalt haben keine intrinsischen, objektiven Bedeutungen. Sie müssen durch politische Entscheidungsträger interpretiert werden, um auf dieser Grundlage auf sie reagieren zu können. Eine Kampagne wie der von den USA proklamierte „Krieg gegen den Terror", der bald nach dem 11. September 2001 begann, erfordert die Konstruktion einer neuen und nicht in Zweifel zu ziehenden „Realität“, in der die Anwendung militärischer Gewalt als normal und vernünftig erscheint. Der amerikanische Präsident Bush und seine Regierung verstanden es am 11. September und in den darauf folgenden Tagen und Wochen, einen Diskurs zu initiieren, der nicht nur die öffentliche Meinung in den USA, sondern auch international bestimmen sollte. Es ging zunächst um die Fragen, was die Anschläge vom 11. September darstellen (,Krieg“" oder „Verbrechen gegen die Menschlichkeit"), wer der Feind ist, welche Art von Bedrohung er verkörpert und wie dieser Bedrohung begegnet werden kann. Es stellte sich dabei in etwa das dar, was Laclau und Mouffe in ihrer Theorie der Hegemonie idealtypisch abbilden, d. h. die Entwicklung eines hegemonialen Diskurses in der Folge des 11. September.

Voraussetzung für diese Entwicklung war eine um sich greifende Sprachlosigkeit infolge der traumatischen Attentate: ,[...] language itself appeared to collapse along with the Twin Towers," so Richard Jackson über den 11. September 2001 (2005: 29). Einige wissenschaftliche Beobachter sprachen gar von einer „Bedeutungsleere“ (,void of meaning“; z. B. Campbell 2002), in deren Kontext sich neue Bedeutungsmuster, wie sie in den Tagen nach dem 11. September von den USA vorgebracht wurden, umso mächtiger entfalten konnten.

Es überrascht, wie schnell die Regierung der USA die entstandene Bedeutungsleere zu füllen vermochte. Nur am 11. September entstand unter dem Eindruck der Katastrophe noch eine kurze Phase des Interpretationsspielraums. Auf der Suche nach einer Erklärung für die Anschläge rang Präsident Bush stundenlang mit der Fassung. „The pictures of airplanes flying into buildings, fires burning, huge structures collapsing, have filled us with disbelief", so Bush in seiner ersten Rede an die Nation (The White House 2001a). Die Ereignisse erschienen durch die permanente Wiederholung im Fernsehen irreal. In den ersten Stunden nach den Anschlägen bestand Unschlüssigkeit darüber, ob dies nun Krieg war oder ein Verbrechen gegen die Menschheit. Justizminister John Ashcroft kündigte kurz nach den Attentaten an: ,We will not tolerate such acts and we will expend every effort 
and devote all necessary resources to bring the people responsible for these crimes to justice“ (U.S. Department of Justice 2001). Und Verteidigungsminister Rumsfeld war zu diesem Zeitpunkt ebenfalls noch nicht auf die später dominierende Terminologie festgelegt. Auf die Frage, ob er die Anschläge als kriegerische Akte bewerte, antwortete er: „What words the lawyers will use to characterize it is for them“ (U.S. Department of Defense 2001a). Erst am Abend gab es erste Anzeichen einer sich ändernden Wortwahl, die die außenpolitische Agenda fortan bestimmen sollte. Die Haltung der USA fasste der Präsident in seiner Rede an die Nation zusammen: „Our military is powerful, and it's prepared. [... A America and our friends and allies join with all those who want peace and security in the world, and we stand together to win the war against terrorism"(The White House 2001a).

Bush wiederholte diese Nachricht an die Welt am nächsten Tag (The White House 2001e), und bereits zwei Tage später waren die Begriffe „Krieg“ und „Kampf gegen den Terror" zur gängigen Amtssprache in Washington geworden. Nach außen fand kaum eine Diskussion der Begriffe statt, obwohl doch durchaus alternative Deutungsmuster möglich gewesen wären. So weist Adam Roberts darauf hin, dass die Angriffe des 11. September auch als „Verbrechen gegen die Menschheit“ (crimes against humanity) kategorisiert werden könnten. Diese umfassten weitreichenden oder systematischen Mord von größeren Menschengruppen (Roberts 2002: 8).

Zumindest handelt es sich nach Ansicht zahlreicher wissenschaftlicher Beobachter um eine neue Art von „Krieg“, die nicht nur jenseits des traditionellen Verständnisses eines instrumentellen Einsatzes militärischer Stärke liegt, sondern auch gängiges jus in bello verletzt. ${ }^{5}$ Von einem „Krieg“ im herkömmlichen Sinne zu sprechen, birgt die Gefahr, die Terroristen nicht als Kriminelle, sondern als Kombattanten nach den Genfer Konventionen zu definieren und ihnen damit einen gewissen Grad an Legitimität zuzuschreiben. Dem Terrorismus den Krieg zu erklären, führt zur Legitimierung krimineller Handlungen als kriegerische Taten. Terroristen werden zu völkerrechtlich anzuerkennenden Soldaten (Howard 2002). Dagegen spricht auch die heterogene und konspirative Struktur terroristischer Organisationen, deren Rechtspersönlichkeit als „,partielles Völkerrechtssubjekt“ völlig unklar wäre (Seidel 2003: 461).

Vor diesem Hintergrund wirkt Rumsfelds Einschränkung nur logisch: „Wir müssen wahrscheinlich ein ganz neues Vokabular erfinden. Was wir vorhaben, unterscheidet sich erheblich von einem Einsatz gegen ein bestimmtes Land in einem bestimmten Zeitrahmen und mit spezifischem Ziel.“6 Der Verteidigungsminister sagte Ende September 2001, es werde keinen D-Day geben, und spielte damit auf die große Landung der Westalliierten in der Normandie 1944 an. Am Ende des Feldzuges gegen den Terrorismus werde es auch keine Kapitulationsurkunde geben, wie sie beispielsweise von Japan zur Beendigung des

5 So das Verständnis von McInnes 2003: 173; ähnlich nüchtern Lawler 2002: 154: „The most powerful sovereign state was attacked from without in a paramilitary fashion that unequivocally constituted a grave breach of the established laws of war.“ Umfassend zum Thema „neue Kriege“ auch Münkler 2002; Howard 2002.

6 „Rumsfeld: Schlag gegen Terrorismus nicht mit Begriff Krieg in Verbindung bringen“, in: Die Welt, 21.09.01. 
Zweiten Weltkriegs im Pazifik unterzeichnet worden sei. Dennoch handele es sich um nichts anderes als einen Krieg, den man gewinnen müsse, so Rumsfeld Ende September 2001: „I don't think about this so much as retaliation or retribution or even justice. I think about it as - you think back to real wars. The goal is victory“ (U.S. Department of Defense 2001c).

Je umfassender die Vorfälle vom 11. September konstruiert wurden, desto umfassender konnte die Antwort ausfallen. So wurde aus den „despicable acts of terror“ (The White House 2001a) vom 11. September in den nächsten Tagen ein ,,act of war“ (The White House 2001b, 2001c). Auf der Grundlage einer Konstruktion der Angriffe als kriegerische Akte formulierte Bush am 15. September unmissverständlich die ultimative Kampfansage an die Urheber der Attentate: ,Those who make war against the United States have chosen their own destruction“" (The White House 2001b).

Einige Beobachter sehen entsprechend einen zunehmend prahlerischen Charakter in den amerikanischen Sprechakten (Murphy 2003; Jackson 2005), der das Feld der klassischen außenpolitischen Wortwahl verlässt und das eigene Land ins Mythische überhöht: „America was targeted for attack because we're the brightest beacon for freedom and opportunity in the world. And no one will keep that light from shining", so der Präsident am 11. September (The White House 2001a). Zu den gebrauchten Stilmitteln gehören Analogien (,Al Qaeda is to terrorism what the mafia is to crime"), Übertreibungen („Al Qaeda wanted to kill all Americans"), visuelle Verstärkungen (Bush mit dem DienstAbzeichen des Polizisten George Howard, der im World Trade Center starb, ,trying to save others“), Anspielungen auf populäre Unterhaltungsshows (the „American hero“) und Historifizierungen (Zweiter Weltkrieg, Pearl Harbor). ${ }^{7}$

Die Konstruktion des „Kriegs gegen den Terror“" weist damit mindestens drei Facetten auf: Erstens wird der Raum für eine Gegenstrategie umso umfassender, je gefährlicher und bedrohlicher der Terrorismus konstruiert wird. Der omnipräsente Terrorismus kann nicht eingedämmt oder abgeschreckt werden, also kann das Ziel nur seine Vernichtung sein. Zweitens dienen Diskurse über „Gefahr“ und „Bedrohung“ zur Konstruktion von Identität; sie ziehen eine unüberschreitbare Linie zwischen ego und alter, Innerem und Äußerem. ${ }^{8}$ Schließlich erzeugen sie drittens Angst in einem Maß, das die Hörerschaft - in diesem Fall die amerikanische und internationale Öffentlichkeit, aber auch politische Entscheidungsträger in anderen Ländern - empfänglicher macht für Maßnahmen aller Art zur Eliminierung der Quellen dieser Angst.

Mit dieser Argumentationsweise soll nicht suggeriert werden, dass Terrorismus keine wirkliche Bedrohung darstellt. Am 11. September konnten die Fernsehzuschauer in aller Welt miterleben, welche realen Gefahren er in sich birgt. Es soll lediglich gezeigt werden, dass Gefahren zu solchen Facetten des sozialen Lebens zählen, die als Bedrohungen interpretiert werden müssen, damit sie intersubjektiv existieren: Gefahren offenbaren sich nicht unabhängig von der Perzeption des Beobachters, sie müssen - in den Worten von Ole

$7 \mathrm{Zu}$ einer umfassenden Analyse der rhetorischen Stilmittel amerikanischer Spitzenpolitiker Jackson 2005.

8 Die Instrumentalisierung von Gefahren zur Identitätsbildung im amerikanischen Sicherheitsdiskurs hat Campbell (1992) exemplarisch analysiert. 
Waever und Barry Buzan - sekuritisiert (d. h. zu einem Sicherheitsproblem gemacht) werden (Buzan/Waever 2003).

Diese Sekuritisierung des 11. September, seine im wahrsten Sinne des Wortes soziale Konstruktion, gelang niemandem besser als dem damaligen stellvertretenden Verteidigungsminister Paul Wolfowitz, der die Vorfälle in der Präsentation der Quadrennial Defense Review am 30. September als Szenario der Sicherheitsbedrohungen des 21. Jahrhunderts konstruierte (U.S. Department of Defense 2001b). Wolfowitz beschrieb die Terroranschläge als „Fenster in unsere Zukunft“ und als „Weckruf“ und forderte vom Kongress wirksame Schritte gegen die terroristische Bedrohung, die in der Zukunft auf die USA warte (U.S. Department of Defense 2001b). Es ist diese Art der Konstruktion, die Mikkel Vedby Rasmussen als ,reflexives Sicherheitsdilemma“ bezeichnet hat (2002: 328). Ereignisse, die in der Zukunft liegen, werden zum Objekt gegenwärtiger Politik. Wolfowitz beschrieb dies in zugespitzter Form: „A future where new enemies visit violence on us in startling ways; a future in which our cities are among the battlefields and our people are among the targets; a future in which more and more adversaries will possess the capability to bring war to the American homeland; a future where the old methods of deterrence are no longer sufficient - and new strategies and capabilities are needed to ensure peace and security" (U.S. Department of Defense 2001b).

Diskurse übten hier als Träger von jeweils gültigem Wissen Macht aus und wurden selber zu einem Machtfaktor, indem sie Verhalten induzierten und neue Diskurse generierten. Die konkrete Konstruktion der Gefahrenquelle bestimmte das Spektrum politischer Handlungsoptionen nach dem 11. September 2001. Auf dieser Grundlage schließt der Diskurs alternative Bedeutungsmuster und Rahmenwerke für Akteursverhalten als illegitim und irrational aus. Gelingt die Vereinnahmung anderer Akteure, kann von einem hegemonialen Diskurs im Sinne von Laclau und Mouffe gesprochen werden. Der Prozess bedeutet dann nichts anderes als Machterzeugung. Damit diese Strategie zum Erfolg führt, appellierte die amerikanische Regierung an die tiefsten Emotionen ihres Publikums. Die Menschen müssen Angst haben, so Paul Wolfowitz am 3. Oktober 2001: „If they [the terrorists] had the capabilities to kill millions of innocent civilians, do any of us believe they would hesitate to do so?" (U.S. Department of Defense 2001b).

Im weiteren Verlauf nahm die Bedrohungskonstruktion an Intensität und Reichweite zu. Die Permanenz des Menetekels wurde fortan zum wichtigsten Pfeiler amerikanischer Regierungspolitik. Im Laufe der Monate, insbesondere nach der Entmachtung der Taliban in Afghanistan, wurde die konkrete terroristische Bedrohung durch Al Kaida und Osama bin Laden indes zusehends begrifflich verwischt und zunehmend in einem Zusammenhang mit den Begriffen „Massenvernichtungswaffen“ und „Schurkenstaaten“ genannt. Der Bezug, wie ihn Präsident Bush am 29. Januar 2002 in seiner Rede über die „Achse des Bösen formulierte, ist eindeutig: „States like these, and their terrorist allies, constitute an axis of evil, arming to threaten the peace of the world. By seeking weapons of mass destruction, these regimes pose a grave and growing danger. They could provide these arms to terrorists, giving them the means to match their hatred." (The White House 2002a)

Der Schachzug, den Bush damit vollzog, war die erneute Einbeziehung des Nationalstaats in seine Argumentation. Obwohl Bush schon am Abend des 11. September ankündigte, 
nicht nur die Terroristen zu bekämpfen, sondern auch jene Staaten, die ihnen Unterschlupf und Schutz gewährten (The White House 2001a), hatte der Schwerpunkt vorher doch auf Individuen und transnational agierenden Organisationen gelegen. Dabei war von Anfang an ein Staat notwendig, um den von den USA geplanten Krieg führen zu können. Der Krieg in Afghanistan richtete sich nicht gegen die Bevölkerung oder Teile derselben („,The United States of America is a friend to the Afghan people“ [The White House $2001 \mathrm{f}$.]), sondern gegen das den Staat repräsentierende Regime.

Nun haben wir es wieder mit Staaten zu tun, denen die Aufmerksamkeit geschenkt werden muss. Dies entspricht einer bestimmten Strömung in der Völkerrechtsliteratur, die fordert, bei einem Anschlag der Größenordnung vom 11. September, der nicht nur die Souveränität der USA, sondern auch die internationale Sicherheit berührt, auch nach der Verantwortlichkeit des Staates zu fragen, der auf seinem Territorium Terroristen Schutz gebietet oder sie anderweitig unterstützt (z. B. Seidel 2003: 460, 463). Da eine Wertung einer Organisation wie Al Kaida als Träger eines Angriffes von außen im Sinne des Völkerrechts schwierig erscheint, wird der Terrorismus als international und nicht mehr lediglich transnational definiert, indem er mit bestimmten Staaten in Verbindung gebracht wird. Aber die Rückkehr zum Staat als Feindobjekt bietet noch eine andere, eher psychologische und auch rechtliche Implikation: Der Gegner wird durch die nationale und internationale Öffentlichkeit nun leichter greifbar. Es ist ein Feind, von dem man eine klar umrissene Vorstellung hat, er ist identifizierbar und besiegbar. Die sicherheitspolitischen Entscheidungen der amerikanischen Regierung bewegten sich zurück auf das vertraute Terrain der klassischen Kriegführung; die Bedrohung wurde völkerrechtlich fassbar, wenn auch leicht widerlegbar: Weder kann die Aggression vom 11. September 2001 eindeutig einem Staat zugeordnet werden - schließlich wurden Al Kaida-Kämpfer auch in Deutschland und sogar den USA selbst ausgebildet -, noch lässt sich konkretisieren, wann von einer Bedrohung der politischen Unabhängigkeit eines Staates gesprochen werden kann.

Dessen ungeachtet zog die amerikanische Regierung die Linie vom 11. September über Afghanistan hinaus bis zum Irak. Von Anfang an wurde der Irak dabei auch mit den Anschlägen vom 11. September in Verbindung gebracht. Am 11. Oktober, vier Tage nach Beginn der Kampfhandlungen in Afghanistan, nannte George W. Bush den Irak erstmals ausdrücklich im Zusammenhang mit dem amerikanischen Kampf gegen den Terror. Die USA würden gegen alle Staaten vorgehen, die bin Laden unterstützten, bekräftigte der amerikanische Präsident. Iraks Präsident Saddam Hussein sei ein „teuflischer Mann“ und werde genau beobachtet. Saddam sei böse und die USA wüssten, dass er Massenvernichtungswaffen entwickle. Deshalb forderten die USA ihn nun auf, internationale Waffenbeobachter ins Land zu lassen (The White House 2001d). Im November 2001 nannte Sicherheitsberaterin Condoleeza Rice ihn in einer Pressekonferenz eine „Bedrohung“, weil „er fest entschlossen ist, Massenvernichtungswaffen in seinen Besitz zu bringen“. Verteidigungsminister Rumsfeld ging noch einen Schritt weiter, indem er erklärte, es gebe „keine Zweifel“ über die „langjährige Verbindung zwischen Al Qaida und dem Irak“.9

Es schließt sich langsam ein Kreis, der das Bild des amerikanischen Denkens nach außen repräsentiert. Dieser Kreis zieht folgende simple aber wirkungsmächtige Argumen-

Beide zit. nach Robert Kagan, Bagdad im Fadenkreuz, in: Die Welt, 28.11.01. 
tationslinie: Der Irak baut bzw. besitzt Massenvernichtungswaffen. Diese Waffen gibt er an Terroristen wie Osama bin Laden weiter. Osama wird diese Waffen in der Zukunft gegen die USA einsetzen. Folglich ist Saddam eine Bedrohung für die Welt. Amerika darf nicht darauf warten, dass diese Bedrohung zur Realität wird; ihr muss vorher begegnet werden. Diese Konstruktionen von Bedrohung, Bestrafung, Selbstverteidigung und Kriegslegitimierung hatten massive Auswirkungen auf die Rekonstruktion des jus ad bellum nach dem 11. September 2001.

\section{Konstruktionen des jus ad bellum}

Die Verstaatlichung des Militärwesens seit dem Westfälischen Frieden 1648 ging in Europa mit einer Verrechtlichung des Krieges einher. Im Rekurs auf klassische philosophische Texte (Cicero, Augustinus, Thomas von Aquin) wurden Theorien vom ,gerechten Krieg“ entworfen, die die Rahmenbedingungen zwischenstaatlicher Gewaltanwendung zum Inhalt hatten. Danach konnte nur dann von einem gerechten Krieg gesprochen werden, wenn auf der einen Seite der Rechtsbrecher identifiziert worden war und die Gegenpartei - den Rechtsbrecher gleichsam bestrafend - in den Krieg eintrat, um das Recht wieder herzustellen (z. B. Grotius 2004; O’Driscoll 2006). Diese Sichtweise implizierte ein asymmetrisches „Recht auf Krieg“ (jus ad bellum), indem die angegriffene Seite quasi das gesamte Recht auf Kriegsführung besaß (Münkler 2002: 110 f.; Daase 2003: 164-168). Im klassischen Völkerrecht war der Krieg als Mittel zur Fortsetzung der Politik mithin nicht als rechtswidrig eingestuft, so lange ein guter Grund dafür bestand (justa causa) und gewisse Formen eingehalten wurden (z. B. eine Kriegserklärung).

Wie im letzten Abschnitt gezeigt wurde, konstruierte die amerikanische Regierung in den Wochen nach dem 11. September aus den „despicable acts of terror” (The White House 2001a) einen gerechten „Krieg gegen den Terror“, der auch in der akademischen Welt Zuspruch fand (insbesondere Elshtain 2003; O'Donovan 2003). Anthony Lang erkennt in der Forderung Bushs, Saddam Hussein für seine Taten zu bestrafen, ein wichtiges Element der Grotius'schen Formulierung vom gerechten Krieg wieder (Lang 2006). Die Idee des gerechten Krieges bringt per definitionem eine Kriminalisierung und mitunter Dämonisierung des Feindes mit sich. Carl Schmitt weist in seiner recht radikalen und inzwischen klassischen Kritik des gerechten Krieges auf die Gefahr hin, dass durch die Idee jeder Gegner gewissermaßen seiner menschlichen Zügen beraubt wird (Schmitt 2003). Damit erhält der gerechte Krieg eine politische Funktion. Genau diesen Prozess sehen einige Beobachter in der amerikanischen Konstruktion des Kriegs gegen den Terror gegeben (Slomp 2006). Der Gerechtigkeitsbegriff (justice) wird einseitig mit Inhalt gefüllt; das Postulat lautet, Gerechtigkeit wiederherzustellen, indem Krieg geführt wird (O'Donovan 2003: 13; zur Kritik O’Driscoll 2006).

Völkerrechtlich bedeutet der Rekurs auf den gerechten Krieg, dass eine Entscheidung über den Einsatz militärischer Mittel in der Interpretation Washingtons allein in der Hand der US-Regierung lag und selbst nach heutigem Völkerrecht kaum angefochten werden konnte, wenn sich die Interpretation einer Notwehr-Situation - Individuelle Selbstverteidigung nach Artikel $51 \mathrm{CVN}$ - durchsetzte. Die in den Jahrzehnten nach dem Zweiten Weltkrieg 
dominierende Lehrmeinung definierte das jus ad bellum mithin allein als Recht von Staaten zur Abwehr einer von außen vorgetragenen Aggression (Seidel 2003: 461; Walzer 1992: 51-59). Entsprechend konstruierte die amerikanische Regierung den „Krieg gegen den Terror" als Akt der Notwehr, als individuelle Selbstverteidigungsstrategie im Sinne der Charta der Vereinten Nationen. Beispielhaft wird diese Haltung in den Worten des stellvertretenden Außenministers Marc Grossman vom 25. Oktober 2001 deutlich: „I believe that Security Council resolution 1368 that was passed on the 12th of September, offers all of the legal basis and requirement that we need, in addition to Article 51 of the United Nations Charter, which is the right of self-defense. And we believe the United States was attacked on the 11th of September and that we have a right of self-defense in this regard" (U.S. Department of State 2001b).

In der Tat scheint der Sicherheitsrat die amerikanische Version des Rechts auf Selbstverteidigung zu übernehmen. Der Rat ,verurteilt einstimmig und in schärfster Weise die schlimmen terroristischen Angriffe des 11. September” und anerkennt „,das naturgegebene Recht auf individuelle und kollektive Selbstverteidigung gemäß der Charta der Vereinten Nationen". ${ }^{10}$ Ohne die amerikanische Kriegsterminologie zu übernehmen, anerkennt er mit einer zweiten Resolution am 28. September nochmals das Recht auf individuelle und kollektive Selbstverteidigung der Vereinigten Staaten. ${ }^{11}$

Es wird in diesem Zusammenhang jedoch häufig übersehen, dass die Auslegung des Rechts auf Selbstverteidigung durch die USA wesentlich weitgehender ist, als durch Artikel 51 der Charta der Vereinten Nationen vorgesehen. Der Interpretationsunterschied bezieht sich auf die Bewertung der Rolle des Sicherheitsrats durch die USA. Die Autorität des Präsidenten wird über diejenige dieses höchsten völkerrechtlichen Entscheidungsgremiums gestellt. Nach den Worten von US-Außenminister Colin Powell Ende September 2001 würden die USA trotz ihres Rechts auf Selbstverteidigung erwägen, ein Mandat der Vereinten Nationen für den militärischen Kampf gegen den Terrorismus zu erhalten. Zwar besäße der US-Präsident die Befugnis, auch ohne die UNO alle für notwendig gehaltenen Maßnahmen zur Selbstverteidigung zu ergreifen, sollte er aber der Meinung sein, es bedürfe weiterer Maßnahmen, dann werde er entscheiden, ob er die Schirmherrschaft der Weltorganisation brauche oder nicht. ${ }^{12}$ Diese Argumentation enthält jedoch einen rechtslogischen Fehler. Für die Ausübung des Selbstverteidigungsrechts nach Art. 51 der Charta ist grundsätzlich keine Zustimmung des Sicherheitsrats nötig; ein Staat muss den Sicherheitsrat jedoch nach der Ausübung dieses naturgegebenen Rechts informieren, worauf der Sicherheitsrat zur Herstellung des Weltfriedens tätig wird und nicht umgekehrt (zur Kritik auch Seidel 2003: 467 ff.).

Dennoch wird man die folgende Militäraktion gegen Afghanistan angesichts der vorliegenden Sicherheitsratsresolutionen, von denen die zweite direkt auf Kapitel VII der

10 S.C. Resolution 1368, U.N. SCOR, 56th Session, 4370th Meeting, at 1, U.N. Document S/RES/1368 (2001).

11 S.C. Resolution 1373, U.N. SCOR, 56th Session, 4370th Meeting, at 1, U.N. Document S/RES/1373 (2001).

12 Vgl. die Aussagen Powells in: Bush bemüht sich um ein UN-Mandat gegen den Terror, in: Die Welt, 28.09.01. 
UN-Charta Bezug nimmt, nicht verurteilen können. Das amerikanische Vorgehen erhält zusätzliche Legitimation, indem der Sicherheitsrat in der Folge die Militärschläge nicht verurteilte und auch die NATO erstmals den Bündnisfall ausrief (NATO Update 2001). Die amerikanische Regierung weist mithin bei jeder Gelegenheit auf den legitimen Charakter der Operation hin: ,Whether we bring our enemies to justice, or bring justice to our enemies, justice will be done“ (The White House 2001c).

Das amerikanische Recht auf Krieg wird zusätzlich aus einer religiös bestimmten Legitimation gewonnen. Die christliche Mission, durch die der Sieg der USA vorher bestimmt ist, betonte der amerikanische Präsident bereits in seiner Rede vor dem Kongress am 20. September: „The course of this conflict is not known, yet its outcome is certain. Freedom and fear, justice and cruelty, have always been at war, and we know that God is not neutral between them" (The White House 2001c). Mit anderen Worten: Gott ist gerecht; die Gerechtigkeit liegt auf der Seite Amerikas, mithin muss Gott auf Seiten der USA stehen. Das „gottlose“ ${ }^{13}$ die Terroristen schützende Afghanistan wird dabei in der gleichen Rede zum ersten offiziellen Ziel militärischer Aktivitäten der Vereinigten Staaten deklariert: „The leadership of al Qaeda has great influence in Afghanistan and supports the Taliban regime in controlling most of that country. In Afghanistan, we see al Qaeda's vision for the world.“ Daher könne das Ultimatum an die politische Führung des Landes nur lauten: „The Taliban must act, and act immediately. They will hand over the terrorists, or they will share in their fate" (The White House 2001c).

Mit dieser Vorgehensweise entzog sich die amerikanische Regierung einer Diskussion der völkerrechtlich höchst umstrittenen Frage, wie viel Staat in einem Angriff durch militärische Kräfte involviert sein muss, damit Gegenmaßnahmen auf der Grundlage des Rechts auf Selbstverteidigung ergriffen werden dürfen. So ging die begriffliche Entwicklung von „Terroranschlag“ zu „Krieg“ einher mit einer Konzentration auf die Taliban Afghanistans als erstem Kriegsziel. Der Terror und Al Kaida spielten zwar mit voranschreitender zeitlicher Entwicklung als Kriegsmotive nach wie vor eine vordergründige Rolle, nun wurden jedoch Staaten die deklarierten Subjekte der Kriegführung. Die Vereinten Nationen spielten in dem unmittelbaren Prozess zur Kriegsentscheidung ebenso wenig eine Rolle wie die NATO. Hier zeigt sich das, was einige Beobachter als Amerikas Unilateralismus bezeichnet haben. „Keine unserer Forderungen wurden von den Taliban erfüllt“, so der Präsident am 7. Oktober, dem Tag der ersten Militärschläge. „Und nun werden sie dafür einen Preis bezahlen“ (The White House 2001 f.). Einmal mehr wird hier das Bestrafungsmotiv deutlich, dass in der Lehre vom gerechten Krieg von jeher eine große Rolle gespielt hat.

Die Idee des Regimesturzes wurde in der ersten Hälfte des Jahres 2002 zu einem der wichtigsten politischen Anliegen des Präsidenten (Rudolf 2003: 270). So erklärte er in seiner Rede vor den Vereinten Nationen am 12. September 2002 im Hinblick auf die Zukunft des Saddam-Regimes nach einer Invasion des Irak durch die USA: „The Security Council resolutions will be enforced, the just demands of peace and security will be met or action will be unavoidable and a regime that has lost its legitimacy will also lose its power"

13 „The terrorists are traitors to their own faith, trying, in effect, to hijack Islam itself “, so Bush am 20.09.01. Zit. nach The White House $2001 \mathrm{f}$. 
(The White House 2002d). Später wurde das Ziel des Regimewechsels in wichtigen Reden amerikanischer Regierungsangehöriger nicht mehr erwähnt. Im Oktober äußerte Bush lediglich noch einmal öffentlich die Hoffnung, dass sich im Falle der Umsetzung aller diesbezüglichen UN-Resolutionen der „Charakter“ des irakischen Staatswesens verändern werde (The White House 2002c).

Ein Kurswechsel war sinnvoll, fehlte es doch beim Ziel des Regimewechsels an einem positivrechtlichen Ausgangspunkt (Bothe 2003: 258). Die Abrüstung des Irak wurde nun zum erklärten Ziel, das vorläufige Instrument dafür waren bedingungslose und ungehinderte Rüstungsinspektionen durch die Vereinten Nationen. Manche Beobachter sehen in diesem Kurswechsel einen brillanten Schachzug des Außenministers Colin Powell (Rudolf 2003: 277; Kaufmann 2004: 29). Zum einen sollte der Krieg völkerrechtlich legitimiert, zum anderen eine breite Koalition gegen Saddam Hussein aufgebaut werden. Alles in allem handelte es sich aber um nicht mehr als einen Scheindiskurs, der sich selbst durch seine inneren Widersprüche enttarnte. Washington hegte eine starke Skepsis gegenüber den Fähigkeiten der Vereinten Nationen, gepaart mit einem tiefen Pessimismus gegenüber jeglicher Gesprächsbereitschaft Bagdads. Auf das Angebot des Irak vom September 2002, nach fast vier Jahren wieder Waffeninspekteure ins Land zu lassen, reagierte die amerikanische Regierung im Rahmen dieses im Herbst 2002 erkennbaren zweigleisigen Musters: Der Präsident sagte während eines Wahlkampfauftritts in Nashville, die Vereinten Nationen müssten nun handeln und beweisen, dass sie mehr seien als eine ,ineffektive Debattiergesellschaft“. Außenminister Powell fügte an, die USA bestünden nun auf einer neuen Resolution des Sicherheitsrats. ${ }^{14}$ In ihrer Reaktion auf das vom irakischen Außenminister Sabri unterbreitete Angebot ließ die US-Regierung verlauten, diese ,enthalte nicht das Versprechen abzurüsten, nicht das Versprechen, Waffeninspekteuren uneingeschränkten Zugang zu gewähren, und nicht das Versprechen, den Stand der Waffenentwicklung offen zu legen. " ${ }^{15}$ Schließlich fasste Paul Wolfowitz im Januar 2003 die Sicht der USA auf die Vereinten Nationen zusammen: Man könne einfach nicht erwarten, dass die UN-Waffeninspekteure einen unkooperativen Irak entwaffneten (U.S. Department of Defense 2003).

Aus den öffentlichen Äußerungen amerikanischer Regierungsangehöriger ließ sich seit dem Herbst 2002 erkennen, dass ein Krieg beschlossene Sache war. Am 2. Dezember beschrieb Pressesprecher Ari Fleischer die Situation wie folgt: Sollte Saddam zugeben, dass er Massenvernichtungswaffen besitze, würde er wieder einmal UN-Resolutionen verletzen; dies müsse harte Konsequenzen haben. Sollte er dagegen behaupten, er besäße keine solchen Waffen, würde er die Welt einmal mehr täuschen, da die amerikanische Regierung Geheimdienstinformationen über ihre Existenz besitze (Woodward 2003: 232). Saddam Hussein saß in einer Falle, aus der es kein Entkommen mehr gab.

Zwei Aspekte spielen im Rahmen des amerikanischen Rechtfertigungsdiskurses eine bedeutende Rolle: einerseits eine gezielte Erzeugung von Angst, wie unmittelbar nach dem 11. September. Sie kam seither kontinuierlich zur Legitimierung außenpolitischer Maßnahmen zum Zuge. Chaim Kaufmann nennt diesen Vorgang ,threat inflation“ (2004). Die Macht der Konstruktionen offenbart sich darin, dass Umfragen zufolge unmittelbar

14 Vgl. ,Washington: Bagdad taktiert. Schröder bietet deutsche Inspekteure an“, in: FAZ, 18.09.02.

15 Zit. nach „Washington: Bagdad taktiert. Schröder bietet deutsche Inspekteure an“, in: FAZ, 18.09.02. 
nach dem 11. September $3 \%$ aller Amerikaner glaubten, Saddam Hussein stehe hinter den Attentaten von Washington, New York und Pennsylvania. Im Januar 2003 waren es bereits $44 \%$, die annahmen, alle oder zumindest die Mehrzahl der Attentäter vom 11. September kämen aus dem Irak, und im September waren es $70 \%$ der Menschen in den USA, die eine Verbindung zwischen den Anschlägen und Saddam Hussein vermuteten (Kaufmann 2004: 20). Die Suggestion wurde verstärkt, indem die Kampagne gegen den Irak von Anfang unter der Überschrift „Krieg gegen den Terror“ verkauft wurde: „Selling the threat“" wurde zu einer der wichtigsten innen- und außenpolitischen Aufgaben Washingtons nach dem 11. September.

Ein zweiter Aspekt, der in diesem Zusammenhang von Bedeutung ist, sind die evangelikalen Glaubensvorstellungen des Präsidenten und wichtiger Mitglieder seiner Regierung. Bush griff in der verbalen Vorbereitung des Krieges gegen den Irak immer wieder auf christliche Konzepte zurück. So stimmte er Amerika und die Welt in seiner Rede zur Lage der Nation vom 28. Januar 2003 auf einen - in seinen Worten - ,präemptiven“ Militärschlag ein. Er beschwörte den „lebendigen Gott“", auf den die Nation stets, wie auch in diesen „entscheidenden Tagen“, ihr Vertrauen setzen könne (The White House 2003b). Dass seine Gründe für die Feldzüge in Afghanistan und im Irak spezifisch christlich-konfessionell waren, lässt sich durch die Analyse des Diskurses nicht direkt belegen, obwohl sich Bush noch 2003 gegenüber Bob Woodward als „Instrument Gottes auf Erden“ bezeichnete (Woodward 2003: 279). Gleichwohl entfalteten seine häufigen rhetorischen Anleihen bei evangelikalen Glaubensüberzeugungen ihre volle öffentliche Wirkungsmacht. Und auf diesem Glaubensfundament entwickelte sich schließlich das amerikanische Völkerrechtsverständnis nach dem 11. September 2001, indem die Verfolgung der Rechtsbrecher nicht durch gerichtliche Instanzen, sondern allein militärisch, durch einen „Bestrafungskrieg“, vollzogen werden soll. Präemptive bzw. ,vorbeugende“ Militärschläge, wenn nötig allein, und kontinuierliche Dominanz werden als die normativen Grundsätze ihrer Verteidigungspolitik deklariert, obwohl das Völkerrecht die Kategorie der Präemption nicht kennt und ein präziser Kriterienkatalog nicht existiert. ${ }^{16}$

Später - insbesondere in der Nationalen Sicherheitsstrategie vom Dezember 2002 wurden die Begriffe Präemption und Prävention wechselweise benutzt. Präemptive sind jedoch von präventiven Militärschlägen zu unterscheiden. Ein Präemptivkrieg fordert die Erfüllung von Kriterien, die die unmittelbare Gefahr der beabsichtigten Aggression eines Staates beurteilen können. Es handelt sich also im Gegensatz zum langfristig angelegten Präventivkrieg um die Ausschaltung einer unmittelbaren Bedrohung. In der Nationalen Sicherheitsstrategie wird daher vor allem offensichtlich, dass man nicht gewillt ist, sich durch gängiges Völkerrecht Handlungsschranken auferlegen zu lassen. Der Grundpfeiler dieser Strategie wird folgendermaßen formuliert: „Verteidigung der Vereinigten Staaten,

16 Die Kontroverse über die Legalität präemptiver Selbstverteidigung war in den letzten Jahrzehnten so stark, dass eine Klärung des Begriffs in wichtigen UN-Resolutionen wie der „Definition of Aggression“ oder der „Declaration on Friendly Relations“ nicht stattfand. Zu einer umfassenden Klärung Schwehm (2006) und Dershowitz, der zur rechtlichen Unsicherheit bemerkt: ,The most disturbing conclusion [...] is that although preventive confinement has always been and will always be practised, no systematic and widely accepted jurisprudence of preventive intervention has ever been developed." (2006: 56) 
des amerikanischen Volkes und unserer nationalen und internationalen Interessen, indem wir Bedrohungen ausmachen und ausschalten, bevor sie unsere Grenzen erreichen. Die Vereinigten Staaten werden sich ständig um die Unterstützung der internationalen Organisationen bemühen, werden aber auch nicht zögern zu handeln, wenn es notwendig werden sollte, unser Recht auf Selbstverteidigung wahrzunehmen, indem wir präemptiv gegen solche Terroristen vorgehen und sie davon abhalten, dass sie unserem Volk und unserem Land Schaden zufügen. [...] Je größer die Bedrohung, desto größer das durch Untätigkeit entstehende Risiko - und desto zwingender das Argument für antizipatorische Selbstverteidigung, selbst wenn Unsicherheit darüber besteht, wann und wo der Feind angreifen wird. Die Vereinigten Staaten werden gegebenenfalls präemptiv handeln, um solche feindlichen Akte unserer Gegner zu vereiteln oder ihnen vorzubeugen“" (The White House 2002b; zu einem Kommentar Krause 2004: 182).

Das Dokument ist weit von einer klaren Definition des Selbstverteidigungsrechts von Staaten entfernt und bietet daher keine Grundlage für die Fortentwicklung des Völkerrechts. Die Neuinterpretation des gängigen jus ad bellum führt lediglich zu einer Ausweitung künftig möglicher Handlungsoptionen der USA. Die Vereinigten Staaten reklamieren für sich in ihrem „Krieg gegen den Terror“ alle militärischen Optionen, ungeachtet der damit verbundenen völkerrechtlichen Probleme. Was zählt, sind Flexibilität und Effizienz der Mittel. Die Normen globaler Sicherheitspolitik werden von Fall von Fall durch den Staat interpretiert, dem in der eigenen Interpretation die größte materielle Verantwortung für die Aufrechterhaltung von Frieden in der Welt zukommt. Zum einen ist dies eine massive Verletzung des „Prinzips der souveränen Gleichheit der Staaten“ (Seidel 2003: 451) mit der Konsequenz einer Rückkehr zum Recht des Stärkeren; zum anderen offenbart sich hier das weiter oben beschriebene „reflexive Sicherheitsdilemma“ in seiner ganzen Tragweite: Ereignisse, die in der Zukunft liegen, werden zum Objekt gegenwärtiger Politik.

Spätestens im Irakkrieg wurde die Strategie einer vorbeugenden Kriegsführung in die Tat umgesetzt. Verteidigungsminister Rumsfeld begründete das Vorgehen in beredter, gleichwohl völkerrechtswidriger Manier mit dem Hinweis, die Abwesenheit eindeutiger Indizien für das Vorhandensein von Massenvernichtungswaffen im Irak biete kein eindeutiges Indiz dafür, dass es dort nicht doch welche gebe (zit. nach Ikenberry 2002: 51). Nach Meinung vieler Beobachter entleert eine solche Aussage jedoch in zynischer Weise Art. 51 CVN seines Inhalts (exemplarisch Ruf 2002; Ikenberry 2002). Es kann hier angesichts des Fehlens eines klaren Bezugspunktes wohl kaum von einem Präventivkrieg gesprochen werden - geschweige denn von einem Präemptivkrieg.

Das traditionelle jus ad bellum wird damit durch die Irak-Invasion der USA in doppelter Weise sinnentleert: erstens durch eine Missachtung des in der Charta der Vereinten Nationen festgeschriebenen Souveränitätsgebots, zweitens durch die Aushöhlung des klassischen gewohnheitsrechtlichen Verständnisses antizipatorischer Selbstverteidigung und des in Artikel 51 der Charta vorgesehenen Rechts auf individuelle und kollektive Selbstverteidigung. Das Kriterium der „unmittelbaren Bedrohung“ muss für diesen Fall der Kriegsführung dermaßen ausgeweitet werden, dass selbst gegenwärtig nur als ,,potenziell“ einzustufende Bedrohungen darunter fallen (Rudolf 2003: 260). Dass es im Irak um eine langfristige Strategie ging, wird sowohl aus Powells Rede vor dem Sicherheitsrat am 
5. Februar 2003 deutlich (U.S. Department of State 2003a) als auch in der als „Ultimatumsrede" bekannten Ansprache des Präsidenten vom 17. März 2003. Wie Peter Rudolf argumentiert, ist dabei vor allem interessant, dass Bush zwei Tage vor Kriegsbeginn nicht eine unmittelbare Gefahr durch den Irak suggerierte (Rudolf 2003: 261). Vielmehr postulierte der Präsident in einer „Global Message“ am 17. März 2003 das Recht für die USA, jedes Land anzugreifen, das irgendwann in der Zukunft zu einer Bedrohung werden könnte (The White House 2003a).

Obwohl sich die USA lange um die Zustimmung des Sicherheitsrates bemühten, wurde die Invasion des Irak am 19. März 2003 nicht durch einen Beschluss des UN-Sicherheitsrats legitimiert, und darin lag letztlich nach Meinung vieler Beobachter ein Völkerrechtsbruch durch die USA. ${ }^{17}$ In ihrem Brief an den Sicherheitsrat zu Beginn der Irak-Invasion bezieht sich die amerikanische Regierung nochmals auf die Resolutionen 678 (1990) und 687 (1991), die der Rat nach der Besetzung Kuwaits durch den Irak verabschiedet hatte, und die eine Gewaltanwendung gegen das Regime Saddam Husseins legitimieren. Völkerrechtsexperten verurteilen jedoch auch diesen Schachzug: Angesichts einer veränderten Bedrohungslage und bereits beschlossener Maßnahmen des Sicherheitsrats zur Beseitigung derselben könne man bei der Verletzung des neuen nicht einfach ein altes Regime wieder aufleben lassen (Bothe 2003: 262 ff.).

Die Vereinten Nationen wurden noch ein weiteres Mal ignoriert, indem der Bitte der UNMOVIC (UN Monitoring, Verification and Inspection Commission) unter der Leitung von Hans Blix nach einem zeitlichen Aufschub für die Inspektoren nicht entsprochen wurde. Als Blix am 14. Februar zum zweiten Mal vor den Sicherheitsrat trat, um dort einen Bericht über die Arbeiten im Irak zu geben, stellte er die von Colin Powell kurz zuvor präsentierten „Beweise“ der US-Regierung deutlich infrage. Man habe die von Powell gezeigten Gebäude und Liegenschaften inspiziert und nichts gefunden. Darüber hinaus könne es als sicher gelten, dass der Irak sein Atomprogramm nicht, wie von den USA behauptet, wieder aufgenommen habe (Freedman 2004: $31 \mathrm{f}$.).

Trotzdem hielten die USA an ihrem Kurs fest. Wie sehr die Vereinigten Staaten sich schon für unantastbar hielten, zeigt ein Aufsehen erregendes Zitat von Vize-Präsident Cheney, in dem er der IAEA explizit Versagen unterstellt: ,[...] if you look at the track record of the International Atomic Energy Agency and this kind of issue, especially where Iraq's concerned, they have consistently underestimated or missed what it was Saddam Hussein was doing. I don't have any reason to believe they're any more valid this time than they've been in the past" (The Vice President's Office 2003a).

Das Verhalten ist von einer schroffen Missachtung der Vereinten Nationen geprägt. „It is the Congress's vote and not the UN's that matters on Capitol Hill“", so beschreibt Kenneth Moss diese Situation (2003: 144). Natürlich sprach in Washington niemand von unilateralem Vorgehen, wurde den USA doch die Unterstützung von so wichtigen Staaten wie Großbritannien, Spanien, Polen, Japan, Australien und anderen zuteil. Dennoch wurden

17 Dies machte auch UN-Gneralsekretär Kofi Annan eine Woche vor der Invasion des Irak deutlich; vgl. „Anporigai de kôgeki“ (Ein Angriff läge außerhalb der Prinzipien des Sicherheitsvertrags [der Charta der Vereinten Nationen], in: Asahi Shinbun, 11.03.03. 
Hegemonie und Transformationswille zu dem Begriffspaar, das die amerikanische Kriegspolitik nach dem 11. September am besten kennzeichnete (Ischinger 2003: 59; Nabers 2005).

\section{Konstruktion, Krieg und Selbstverteidigung}

Aus den traumatischen Ereignissen des 11. September und dem damit einhergehenden Vertrauensverlust in die Sicherheit, Verlässlichkeit und Gerechtigkeit der Welt resultierte schon bald eine enorme Dynamik des offiziellen sicherheitspolitischen Diskurses, ein Druck zur Definition der Vorkommnisse, zur Benennung des Feindes und zur politischen Aktion. Die Ereignisse des 11. September besitzen keine objektive Bedeutung; sie wurden erst in den folgenden Tagen und Wochen diskursiv konstruiert. Es wurden dabei völlig neue Begriffe gewählt oder alte Konzepte in einen neuen Kontext gesetzt. Der Konstruktion internationaler Normen nach dem 11. September ist dabei eine Spannung immanent, die durch den amerikanisch-dominierten Diskurs behelfsmäßig überbrückt werden soll: Es herrscht Krieg, aber der Krieg unterscheidet sich von allem da gewesenen.

Der „Krieg gegen den Terror“ ist ein diskursives Projekt, das in bestimmte institutionalisierte sicherheitspolitische Handlungen mündet. Die Analyse von Diskursen gibt uns Aufschluss darüber, wie bestimmte Ideen sich gegenüber anderen durchsetzen, wie Normen verhandelt, gestärkt, verletzt und reformuliert werden, wie Identität konstruiert und Macht legitimiert wird, und wie sich bestimmte politische Handlungsweisen gegenüber anderen, alternativen Handlungen durchsetzen. Sie zeigt, wie Hegemonie durch die Formulierung und Reformulierung von Ideen entsteht. Sprache ist niemals neutral; sie beschreibt die Welt nicht lediglich, sie erschafft die Welt durch bestimmte Realitäts- und Aussageannahmen in unseren Köpfen. Die Rhetorik der Bush-Regierung dokumentiert in eindringlicher Weise die perlokutionäre Kraft des Kriegsbegriffs. Wenn illokutionäre Akte, wie hier sehr anschaulich geschehen, eine Rolle in einem teleologischen Handlungskontext übernehmen, werden sie zu perlokutionären Akten (Searle 1969: 25). Die Ereignisse des 11. September mussten zunächst diskursiv konstruiert werden, um einen politischen Sinn zu ergeben. Gelingt die Vereinnahmung anderer Akteure durch bestimmte Wertannahmen, kann von einem hegemonialen Diskurs im Sinne von Laclau und Mouffe gesprochen werden. Insofern ist diese Krise eine Quelle von Macht, da sie völlig neue Interpretationsspielräume eröffnet.

Dies hat erhebliche Konsequenzen für die politische Praxis. Die Sprache des „Kriegs gegen den Terror“ hat die Funktion, die Praxis des „Kriegs gegen den Terror“ zu normalisieren und zu legitimieren. Partikulare, strikt auf den 11. September bezogene Diskurse nehmen die Tendenz von universellen Diskursen über den Zustand der globalen Sicherheit an. Je weitgreifender ein hegemonialer Diskurs ist, desto normaler erscheinen seine Inhalte einer großen Gruppe von Zuhörern. Christliches Vokabular, Bestrafungslogik und die suggestive Kraft der Idee eines ,gerechten Kriegs“, gekoppelt mit der Erzeugung von Angst als politischem Instrument, normalisieren den Krieg und reduzieren die strengen Erwartungen des jus in bello.

Zur erfolgreichen Hegemonialisierung infolge des hier konzeptualisierten traumatischen Ereignisses „11. September 2001“ war zunächst die Benennung bestimmter Personen als 
Urheber der Krise notwendig (zu diesen Zusammenhängen auch Brockhaus 2002). Reduktion von Komplexität war gefordert. Die Einsicht in die Abstraktheit der Wirkungsgesetze eines Ereignisses wie des11. Septembers geht vielen Menschen verloren. Nur aus diesem Grund war die frühe Benennung von Osama bin Laden als Feindbild notwendig. Angst und Trauma erzeugen Kollektivität. Das „Eigene“ wird homogenisiert, das „Andere“ ausgegrenzt. Sicherheit wird dabei in einer solchen Krise durch die residuale Verbindung mit alten Bedeutungsmustern und Institutionen wieder hergestellt. Die Anschläge des 11. September werden als Zeichen des Krieges gedeutet, der mit einem militärischen Angriff auf einen Staat (Afghanistan, „Achse des Bösen“) beantwortet werden kann. Damit werden die Attentate als bekanntes Phänomen dargestellt, das mit den Mitteln der klassischen Kriegsführung gelöst werden kann. Natürlich liegt das Gesetz des Handelns allein in den eigenen Händen. Nur so kann ein Trauma überwunden werden. Warten zu müssen auf die langsam vonstatten gehende strafrechtliche Verfolgung der Täter wäre unerträglich; nur die militärische Reaktion gibt der Volksseele das benötigte Selbstvertrauen zurück, indem sie Stärke und Unbesiegbarkeit repräsentiert. Die Produktion eines Außenfeindes spielt in diesem Kontext eine bedeutende Rolle. Der terroristische Feind kommt zwar auch aus dem Inneren der westlichen Gesellschaft und hat sich seine tödlichen Techniken in Ländern wie Deutschland und den USA angeeignet. Zur Überwindung des Traumas musste jedoch glaubhaft gemacht werden, dass der Täter von außen kam und als solcher bekämpft werden konnte.

Was damit deutlich wird, ist der Zusammenhang von Diskurs und Macht auch für die Fortentwicklung des Völkerrechts. Dabei ist genau zu beobachten, wer die Interpretationshoheit in Bezug auf einen bestimmten Gegenstand besitzt. Die USA legten nach dem 11. September indes keine klaren Konzepte von Selbstverteidigung, Präemption und Prävention vor. Die Kritik an der gängigen Auslegung des Selbstverteidigungsrechts wurde nicht in eine positive Rechtsbehauptung umgesetzt. Stattdessen wurde der in der Tradition der philosophischen Lehre vom Krieg zentrale Begriff „Gerechtigkeit“" (justice) in unterschiedlicher Weise moralisch, evangelikal oder juristisch gebraucht und erzeugte eine große Unsicherheit in der völkerrechtlichen Diskussion. Die Vermischung der drei Sphären im amerikanischen Diskurs verzerrte die Konturen des Konzepts der Selbstverteidigung, ohne zu einer Neuformulierung wichtiger rechtlicher Kategorien zu führen. Diese Verzerrung des rechtlichen Diskurses ist am Ende der Grund für das Scheitern der Hegemonialstrategie der USA nach dem 11. September 2001.

Natürlich wurde das Gewaltverbot durch die Kriege in Afghanistan und im Irak einem erheblichen Druck ausgesetzt, der zur Entstehung ,,spontanen Gewohnheitsrechts“ führen könnte (Seidel 2003: 461; Bothe 2003). Doch fehlt für diese völkerrechtliche Weiterentwicklung als wichtigste Voraussetzung die uneingeschränkte Unterstützung der Staatengemeinschaft wie auch der Völkerrechtswissenschaft. Der Tenor des Schrifttums seit dem 11. September 2001 geht dahin, das Gewaltverbot der UN-Charta als zivilisatorische Errungenschaft des 20. Jahrhunderts anzusehen, deren Schutz der gesamten Staatengemeinschaft mit dem UN-Sicherheitsrat als Koordinierungsgremium zu übertragen ist. Die Kriege gegen Afghanistan und Irak können daher nicht als Präzedenzfälle für ein neues Selbstverteidigungsrecht angesehen werden. Die Wiederherstellung des Friedens jeweils 
in die Verantwortung des Staates zu geben, der selbst Opfer eines Angriffs geworden ist, birgt unabsehbare Risiken für die globale Sicherheit - auch wenn es sich um den Staat mit den weltweit größten militärischen Kapazitäten handelt.

\section{Literatur}

Bothe, Michael, 2003: Der Irak-Krieg und das völkerrechtliche Gewaltverbot, in: Archiv des Völkerrechts 41, 255-271.

Brockhaus, Gudrun, 2002: Die Reparatur der Ohnmacht. Zur Psychologie der politischen Reaktionen auf den 11. September 2001, in: Politische Studien 386, 42-60.

Buzan, Barry/Waever, Ole, 2003: Regions and Powers. The Structure of International Security, Cambridge.

Campbell, David, 2002: Time is broken. The return of the past in the response to September 11, in: Theory and Event 5 (4), http://muse.jhu.edu/journals/theory_and_event/v005/ 5.4campbell.html (Stand: 22.01.05).

Daase, Christopher, 2003: Krieg und politische Gewalt: Konzeptionelle Innovation und theoretischer Fortschritt, in: Gunther Hellmann/Klaus Dieter Wolf/Michael Zürn (Hrsg.), Die neuen Internationalen Beziehungen. Forschungsstand und Perspektiven in Deutschland, Baden-Baden.

Dershowitz, Alan M., 2006: Preemption. A Knife that Cuts Both Ways, New York.

Deutsches Rotes Kreuz, 1988: Die Genfer Rotkreuz-Abkommen vom 12. August 1949 und die beiden Zusatzprotokolle vom 8. Juni 1977. Mit einer Einführung von Anton Schlögel, Bonn.

Diez, Thomas, 1999: ,Speaking Europe“: The Politics of Integration Discourse, in: Journal of European Public Policy 6 (4), 598-613.

Doty, Roxanne L., 1996: Imperial Encounters, Minneapolis.

Edkins, Jenny, 2002: Forget Trauma? Responses to September 11, in: International Relations 16 (2), 243-256.

Elshtain, Jean Bethke, 2003: Just War against Terror: the Burden of American Power in a Violent World, New York.

Fairclough, Norman, 1989: Language and Power, London.

Fairclough, Norman, 1992: Discourse and Social Change, Cambridge.

Fairclough, Norman, 1999: Linguistic and Intertextual Analysis within Discourse Analysis, in: Adam Jaworski/Nikolas Coupland (Hrsg.), The Discourse Reader, London/New York, 181-211.

Fairclough, Norman, 2003: Analysing Discourse. Textual analysis for social research, London/New York.

Foucault, Michel, 1978: Dispositive der Macht, Berlin.

Freedman, Lawrence, 2004: War in Iraq: Selling the Threat, in: Survival 46 (2), 7-50.

Grotius, Hugo, 2004 [1625]: Vom Recht des Krieges, in: Michael Czelinski/Jürgen Stenzel (Hrsg.), Krieg. Philosophische Texte von der Antike bis zur Gegenwart, Stuttgart.

Höhne, Thomas, 2001: Alles konstruiert, oder was? Über den Zusammenhang von Kon- 
struktivismus und empirischer Forschung, in: Johannes Angermüller/Katharina Bunzmann/Martin Nonhoff (Hrsg.), Diskursanalyse: Theorien, Methoden, Anwendungen, Hamburg, 23-35.

Howard, Michael, 2002: What's in a Name? How to Fight Terrorism, in: Foreign Affairs, January/February, 8-13.

Ikenberry, G. John, 2002: America's Imperial Ambition, in: Foreign Affairs, September/ October, 44-60.

Ischinger, Wolfgang, 2003: Durch Wandel zu Stabilität. Anmerkungen zur Zukunft der transatlantischen Beziehungen, in: Internationale Politik, Heft 9, 57-65.

Jackson, Richard, 2005: Writing the War on Terrorism: Language, Politics and Counterterrorism, Manchester.

Kaufmann, Chaim, 2004: Threat Inflation and the Failure of the Marketplace of Ideas: The Selling of the Iraq War, in: International Security 29 (1), 5-48.

Krause, Joachim, 2004: Die Krise der westlichen Allianz und die Krise des Multilateralismus, in: Sicherheit + Frieden 22 (4), 179-190.

Laclau, Ernesto, 1977: Politics and Ideology in Marxist Theory: Capitalism, Fascism, Populism, London.

Laclau, Ernesto, 1990: New Reflections of the Revolution of Our Time, London.

Laclau, Ernesto (Hrsg.), 1994: The Making of Political Identities, London.

Laclau, Ernesto/Mouffe, Chantal, 1985: Hegemony and Socialist Strategy. Towards a Radical Democratic Politics, London.

Lang, Anthony F., 2006: Punitive Justifications or Just Punishment? An Ethical Reading of Coercive Diplomacy, in: Cambridge Review of International Affairs 19 (3), 389-403.

Larsen, Henrik, 1997: Foreign Policy and Discourse Analysis. France, Britain and Europe, London/New York.

Lawler, Peter, 2002: The „Good War” after September 11, in: Government and Opposition 37 (2), 151-172.

Mayer, Peter, 2003: Die „Epistemologie“ der Internationalen Beziehungen: Anmerkungen zum Stand der „Dritten Debatte“, in: Gunther Hellmann/Klaus Dieter Wolf/Michael Zürn (Hrsg.), Die neuen Internationalen Beziehungen. Forschungsstand und Perspektiven in Deutschland, Baden-Baden, 47-98.

McInnes, Colin, 2003: A different kind of war? September 11 and the United States' Afghan War, in: Review of International Studies 29, 165-184.

Milliken, Jennifer, 1999: The Study of Discourse in International Relations: A Critique of Research Methods, in: European Journal of International Relations 5 (2), 225-254.

Moss, Kenneth B., 2003: Reasserting American Exceptionalism - Confronting the World: The National Security Strategy of the Bush Administration, in: Internationale Politik und Gesellschaft, Heft 3, 135-155.

Münkler, Herfried, 2002: Die neuen Kriege, Reinbek.

Murphy, John, 2003: „Our Mission and Our Moment“: George W. Bush and September 11, in: Rhetoric and Public Affairs 6 (4), 607-632.

Nabers, Dirk, 2005: Allianz gegen den Terror. Japan, Deutschland und die USA, Wiesbaden. 
NATO Update, 2001: Invocation of Article 5 confirmed, 2 October 2001, http://www.nato. int/docu/update/2001/1001/e1002a.htm (Stand: 13.07.01).

O'Donovan, Oliver, 2003: The Just War Revisited, Cambridge.

O'Driscoll, Cian, 2006: Re-negotiating the Just War: The Invasion of Iraq and Punitive War, in: Cambridge Review of International Affairs 19 (3), 405-420.

Rasmussen, Mikkel Vedby, 2002: „A Parallel Globalization of Terror“: 9-11, Security and Globalization, in: Cooperation and Conflict 37 (3), 323-349.

Roberts, Adam, 2002: Counter-Terrorism, Armed Force and the Laws of War, in: Survival 44 (1), 7-32.

Rudolf, Peter, 2003: Der 11. September, die Neuorientierung amerikanischer Außenpolitik und der Krieg gegen Irak, in: ZfP NF 50 (3), 257-280.

Ruf, Werner, 2002: „Die Welt aus den Fugen? Eine neue Welt(un)ordnung von US Gnaden“, in: Friedenspolitischer Ratschlag, http://www.uni-kassel.de/fb10/frieden/rat/2002/ruf. html (Stand: 10.08.04).

Schmitt, Carl, 2003 [1938]: Die Wendung zum diskriminierenden Kriegsbegriff, Berlin.

Schwehm, Johannes, 2006: Präventive Selbstverteidigung als Antwort? Rekonstruktion eines Weltbildes, in: Wissenschaftszentrum für Sozialforschung, Berlin (SP IV 2006302).

Searle, John R., 1969: Speech Acts: An Essay in the Philosophy of Language, Cambridge.

Seidel, Gerd, 2003: Quo vadis Völkerrecht?, in: Archiv des Völkerrechts 41, 449-483.

Seidl-Hohenfeldern, Ignaz, 1987: Völkerrecht, Berlin u. a. (6. Aufl.).

Slomp, Gabriella, 2006: Carl Schmitt's Five Arguments against the Idea of Just War, in: Cambridge Review of International Affairs 19 (3), 435-447.

Smith, Anna Marie, 1998: Laclau and Mouffe. The radical democratic imaginary, London/ New York.

Thürer, Daniel, 2003: Irak-Krise: Anstoß zu einem Überdenken der völkerrechtlichen Quellenlehre?, in: Archiv des Völkerrechts 41, 314-326.

Titscher, Stefan/Meyer, Michael/Wodak, Ruth/Vetter, Eva, 2000: Methods of Text and Discourse Analysis, London/Thousand Oaks.

Tomuschat, Christian, 2001: Der 11. September 2001 und seine rechtlichen Konsequenzen, in: Europäische Grundrechte-Zeitschrift, 535-545.

U.S. Department of Defense, 2001a: DoD News Briefing on Pentagon Attack, Secretary of Defense Donald H. Rumsfeld, September 11, 2001, http://www.defenselink.mil/transcripts/2001/t09112001_t0911sd.html (Stand: 20.04.04).

U.S. Department of Defense, 2001b: Prepared Statement for the House and Senate Armed Services Committees: „Building a Military for the 21st Century“, By Deputy Secretary of Defense Paul Wolfowitz, October 3 and 4, 2001, http://www.defenselink.mil/ speeches/2001/s20011003-depsecdef.html (Stand: 20.11.03).

U.S. Department of Defense, 2001c: News Transcript: Secretary Rumsfeld Interview with NBC „Meet the Press“ with host Tim Russert, 30 September 2001, http://www.defenselink.mil/transcripts/2001/t09302001_t0930sd.html (Stand: 17.10.06).

U.S. Department of Defense, 2003: Deputy Secretary Paul Wolfowitz Policy Address on Iraqi Disarmament, Council on Foreign Relations, New York, January 23, 2003, http:// 
www.cfr.org/publication.html?id=5454 (Stand: 18.04.07).

U.S. Department of Justice, 2001: Remarks of Attorney General John Ashcroft, Press Briefing FBI Headquarters, September 11, 2001, http://www.usdoj.gov/archive/ag/ speeches/2001/agcrisisremarks.htm (Stand: 03.02.01).

U.S. Department of State, 2001a: Remarks to the National Foreign Policy Conference for Leaders of Nongovernmental Organizations, Secretary Colin L. Powell, October 26, 2001, http://www.state.gov/secretary/former/powell/remarks/2001/5762.htm (Stand: 20.03.04).

U.S. Department of State, 2001b: Transcript: Grossman on War against Terrorism, October 25, 2001, http://usembassy.state.gov/islamabad/wwwh01102502.html (Stand: 20.07.03).

U.S. Department of State, 2003: Remarks to the United Nations Security Council, Secretary Colin L. Powell, New York City, February 5, 2003, http://www.informationclearinghouse.info/article3710.htm (Stand: 20.11.03).

The Vice President's Office, 2003: Vice President Cheney's Interview with NBC, Meet the Press, March 16, 2003, Transcript, http://www.mtholyoke.edu/acad/intrel/bush/ cheneymeetthepress.htm(Stand: 07.04.04).

Waever, Ole, 1998: Explaining Europe by Decoding Discourses, in: Anders Wivel (Hrsg.), Explaining European Integration, Copenhagen, 100-146.

Walzer, Michael, 1992: Just and Unjust Wars: A Moral Argument with Historical Illustrations, New York.

The White House, 2001a: Statement by the President in his Address to the Nation, September 11, 2001, http://www.whitehouse.gov/news/releases/2001/09/20010911-16.html (Stand: 15.12.03).

The White House, 2001b: Radio Address of the President to the Nation, September 15, 2001, http://www.whitehouse.gov/news/releases/2001/09/20010915.html (Stand: 23.11.03.).

The White House, 2001c: Address of the President to a Joint Session of Congress and the American People, September 20, 2001, http://www.whitehouse.gov/news/releases/2001/09/20010920-8.html (Stand: 20.11.03).

The White House, 2001d: President Holds Prime Time News Conference, October 11, 2001, http://www.whitehouse.gov/news/releases/2001/10/20011011-7.html (Stand: 20.09.03).

The White House, 2001e: Remarks by the President In Photo Opportunity with the National Security Team, September 12, 2001, http://www.whitehouse.gov/news/releases/2001/09/20010912-4.html (Stand: 20.11.03).

The White House, 2001f: Presidential Address to the Nation, October 7, 2001, http://www. whitehouse.gov/news/releases/2001/10/20011007-8.html (Stand: 10.01.03).

The White House, 2002a: President Delivers State of the Union Address, January 29, 2002, http://www.whitehouse.gov/news/releases/2002/01/20020129-11.html (Stand: 20.12.03.).

The White House, 2002b: Remarks by the President on Iraq, Cincinnati, Ohio, October 7, 2002, http://www.whitehouse.gov/news/releases/2002/10/20021007-8.html (Stand: 10.08.03). 
The White House, 2002c: President Bush Outlines Iraqi Threat, October 7, 2002, http:// www.whitehouse.gov/news/releases/2002/10/20021007-8.html (Stand: 16.08.03).

The White House, 2002d: President's Remarks at the United Nations General Assembly, September 12, 2002, http://www.whitehouse.gov/news/releases/2002/09/200209121.html (Stand: 20.04.03).

The White House, 2003a: Global Message, President George W. Bush, March 17, 2003, http:// www.whitehouse.gov/news/releases/2003/03/20030317-10.html (Stand: 10.08.04).

The White House, 2003b: SOTU Excerpts on Defending Peace \& Security at Home, January 28, 2003, http://www.whitehouse.gov/news/releases/2003/01/20030128-22.html (Stand: 22.12.03).

Woodward, Bob, 2003: Plan of Attack, New York.

Korrespondenzanschrift:

PD Dr. Dirk Nabers

GIGA German Institute of Global and Area Studies

Institut für Asien-Studien

Rothenbaumchaussee 32

20148 Hamburg

E-Mail: nabers@giga-hamburg.de

\section{Forschungsstand Politikwissenschaft}

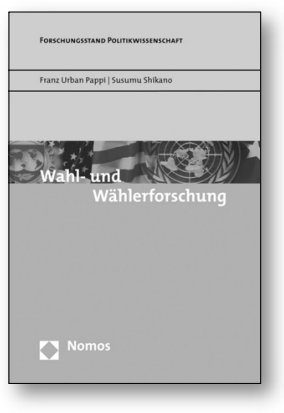

\section{Wahl- und Wählerforschung}

Von Franz Urban Pappi und Susumu Shikano

2007, 250 S., brosch., 19,90 €, ISBN 978-3-8329-2345-7

Der Band legt den Stand der Forschung im Bereich der Wahl- und Wählerforschung dar. Dabei werden zentrale Diskurse analysiert, die Ergebnisse herausgefiltert und auf ihre Bedeutung für die gegenwärtige Forschung untersucht. Der Band ist ein ideales Nachschlagewerk für die Wahlforschung und für Interessenten, die sich schnell und grundlegend in das Themengebiet einarbeiten wollen. 